\title{
Comparison of the thickness of the gastrocnemius through ultrasonography during heel-drop exercise performance
}

\author{
Dan-Bee Gal ${ }^{a}$, Su-Young Lee ${ }^{b}$ \\ aPhysical Therapy Room, Good Morning Hospital, Pyeongtaek, Republic of Korea \\ bepartment of Physical Therapy, Division of Health Science, Baekseok University, Cheonan, Republic of Korea
}

\begin{abstract}
Objective: This study was aimed to compare the thickness and pennation angle of gastrocnemius through ultrasonography during the heel-drop exercise on ankle dorsiflexion angle.

Design: Cross-sectional study.

Methods: Nineteen normal adults in their 20 s had voluntarily participated in this study. All subjects performed the ankle heel-drop exercise with ankle dorsiflexed to $0^{\circ}, 10^{\circ}$, and $20^{\circ}$ : heel-drop exercise with ankle dorsiflexed to $0^{\circ}$ was executed on floor-level, heel-drop exercise with ankle dorsiflexed to $10^{\circ}$ on a wooden-block of $2.3 \mathrm{~cm}$ in height, and heel-drop exercise with ankle dorsiflexed to $20^{\circ}$ on a wooden-block of $5.5 \mathrm{~cm}$ in height. In each regimen, the subjects completed a session of 100 heel-drop exercises ( 10 repetitions $\times 10$ sets; with 30 seconds rest following each set; with 24 hours rest following each exercise). Before and immediately after each heel-drop exercise, the thickness and pennation angle of gastrocnemius were measured using an ultrasonography.
\end{abstract}

Results: After the performance of the heel drop exercises with ankle dorsiflexed to $0^{\circ}, 10^{\circ}$, and $20^{\circ}$, the thickness of the gastrocnemius was significantly higher than pre-exercise $(p<0.05)$, and furthermore heel-drop exercise with ankle dorsiflexed to $10^{\circ}$ was significantly higher than exercise with the ankle dorsiflexed to $0^{\circ}(p<0.05)$. However, as for the pennation angle of the gastrocnemius, there were no significant changes after each heel-drop exercise.

Conclusions: This finding suggest that the heel-drop exercise with ankle dorsiflexed to $0^{\circ}, 10^{\circ}$, and $20^{\circ}$ is effective on the strengthening of the gastrocnemius. Furthermore, the heel-drop exercise with the ankle dorsiflexed to $10^{\circ}$ is more effective than with the ankle dorsiflexed to $0^{\circ}$.

Key Words: Ankle joint, Gastrocnemius muscle, Muscle strength, Ultrasonography

\section{Introduction}

Falling is defined as the process of falling or landing on the floor due to sudden, involuntary changes in posture [1]. Falls are considered as one of the most common injuries that can occur to humans from the day they are born to when they become an elderly adult. Worldwide, it is reported annually that $30 \%$ of falls occur amongst those who are over the age of 65 years [2]. It has been stated that lower extremity strength and balance are needed to prevent falls [3], and Wolfson et al. [4] has reported that decreased balance was related to weak ankle muscle strength. Lamontagne et al. [5] has found that decreased control of the ankle decreases gait and balance abilities, which causes an increase in fall risk. The body has the ability to regain standing balance by using the ankle strategy, hip strategy, or both strategies [6]. The ankle strategy is used to counteract against a small amount of sway [7], and is usually the first strategy to be used for recovering standing balance [6].

The ability to maintain balance during anterior and posterior sway is dependent upon the muscle activation of the anterior tibialis and gastrocnemius medialis muscle [8].

Received: 21 April, 2016 Revised: 5 June, 2016 Accepted: 8 June, 2016

Corresponding author: Su-Young Lee

Department of Physical Therapy, Division of Health Science, Baekseok University, 76 Munam-ro, Dongnam-gu, Cheonan 31065 , Republic of Korea Tel: 82-41-550-2546 Fax: 82-41-550-2827 E-mail: rosei118@bu.ac.kr

(c) This is an Open-Access article distributed under the terms of the Creative Commons Attribution Non-Commercial License (http://creativecommons.org/licens es/by-nc/4.0) which permits unrestricted non-commercial use, distribution, and reproduction in any medium, provided the original work is properly cited.

Copyright $@ 2016$ Korean Academy of Physical Therapy Rehabilitation Science 
Therefore, it is necessary to increase ankle joint muscle strength and range of motion. Choi et al. [9] conducted a study on the use of elastic band for resistive exercises and cushion pads for equilibrium exercises. The results showed significant improvement in balance, gait, and muscle endurance. Son [10] conducted a study involving stroke patients performing ankle dorsiflexion strengthening exercises for balance and Lee [11] investigated the effects of using a stationary bike, ankle stretches, and progressive resistive exercises with elastic bands. Thus several studies examining the effects of implementing ankle exercise programs on balance and gait ability has been conducted.

Therefore, although there are many studies on developing rehabilitation programs for Achilles tendinopathy, the purpose of this study was to investigate for an appropriate heel-drop exercises method for reducing Achilles tendon stiffness and improving gastrocnemius strength, as well as its effects on the gastrocnemius muscle tissue in normal, healthy adults.

Alfredson et al. [12] was the first to introduce the heel-drop exercise method. High-intensity eccentric exercises for chronic mid-portion Achilles tendinopathy have been proven to be an effective rehabilitation method [13].

Nevertheless, research focusing on heel-drop exercises and ankle strategy for gastrocnemius muscle strengthening are incomplete, and studies examining for gastrocnemius muscle elongation during heel-drop exercise performance starting from $0^{\circ}$ ankle dorsiflexion are continuously being conducted. Also, the most effective position for heel-drop exercise performance has not yet been fully established. Although Jeong et al. [14] conducted a study involving heel-drop exercise performance depending on gastrocnemius muscle elongation it mainly focuses on the effects of strain and stiffness of the gastrocnemius aponeurosis-tendon.

Diagnostic ultrasound is a device used to evaluate the structural stability and morphological characteristics of the soft tissue in static and dynamic conditions, such as changes in muscle thickness and muscle fiber cross-sectional area, as well as assess the changes in muscle density through visual imaging [15]. In addition, the cross-sectional area and pennation angle increases with decreasing length of the muscle fiber, which are both important factors in predicting the muscle tension [16].

Therefore, the purpose of this study was to investigate the effects of performing heel-drop exercises with various degrees of ankle dorsiflexion on gastrocnemius muscle thick- ness and pennation angle in normal, healthy subjects in their twenties, as well as to discover which dorsiflexion angle is the most effective.

\section{Methods}

\section{Subjects}

Nineteen participants in their twenties from B University of Chungcheongnam-do Cheonan-si had volunteered to participate in this study. The participants had an average age of 22.4 years, average weight of $62.5 \mathrm{~kg}$, and an average height of $166.3 \mathrm{~cm}$ (Table 1). Participants who exhibited normal ankle dorsiflexion, plantarflexion, no history of ankle injury, no lower extremity injuries, and the ability to perform the heel-drop were included in this study. Participants with neurological or orthopedic ailments of the lower extremities, had regularly received over 3 months of strength training, and wore high heels regularly were excluded from the study. The participants had voluntarily agreed to participate in the study after they had been informed of the study objectives and procedures.

\section{Equipment}

\section{Ultrasonography}

The LOGIQ P6 PRO diagnostic ultrasound device (GE Inc., Waukesha, WI, USA) was used to assess the thickness and pennation angle of the gastrocnemius muscle. All ultrasound scans were performed using B mode, $10 \mathrm{MHz}$ central frequency, $60 \mathrm{~Hz}$ sampling frequency, with a linear transducer of $50 \%$ gain, and the muscle thickness was measured using the built-in caliper of the ultrasound [17].

\section{Heel-drop exercise protocol}

Each subject performed the heel-drop exercises in various degrees of ankle flexion $\left(0^{\circ}, 10^{\circ}, 20^{\circ}\right)$ in random order, and the diagnostic ultrasound imaging device was used to examine the calf muscle thickness immediately after exercise. Although this study was based on the Alfredson et al.'s [12] protocol, which included performing a frequency of $180 \mathrm{ec}-$

Table 1. General characteristics of subjects $(\mathrm{N}=19)$

\begin{tabular}{lcrc}
\hline & Male $(\mathrm{n}=10)$ & Female $(\mathrm{n}=9)$ & \multicolumn{1}{c}{ Total } \\
\hline Age $(\mathrm{y})$ & $21.9(2.8)$ & $23(1.1)$ & $22.4(2.2)$ \\
Height $(\mathrm{cm})$ & $171.7(4.1)$ & $160.4(6.3)$ & $166.3(7.7)$ \\
Weight $(\mathrm{kg})$ & $70(11.2)$ & $54.2(6.3)$ & $62.5(12.1)$ \\
\hline
\end{tabular}

Values are presented as mean (SD). 
centric heel drops per day, the protocol has been modified to a frequency reported "as tolerated" by the subjects due to the possible development of discomfort, which is based on Stevens and Tan [13] has reported findings that there was no significant difference in improvement according to the frequency of 180 verses "as tolerated".

\section{$0^{\circ}$ ankle dorsiflexion heel-drop exercise}

After a goniometer was used to confirm $0^{\circ}$ of ankle dorsiflexion in barefoot conditions, subjects were instructed to maximally raise the soles of their feet by standing on their tip-toes, and then to return back into $0^{\circ}$ of ankle dorsiflexion, which was considered as one heel-drop (Figure 1A).

\section{$10^{\circ}$ ankle dorsiflexion heel-drop exercise}

To set the ankle in $10^{\circ}$ of dorsiflexion, $1 / 3$ of the subject's phalanges and metatarsal bones were placed onto a wooden block that was $2.3 \mathrm{~cm}$ in height. After a goniometer was used to confirm $10^{\circ}$ of dorsiflexion, subjects were instructed to maximally raise the soles of their feet by standing on their tip-toes, and then to return back into $10^{\circ}$ of ankle dorsiflexion, which was considered as one heel-drop (Figure 1B).

\section{$20^{\circ}$ ankle dorsiflexion heel-drop exercise}

To set the ankle in $20^{\circ}$ of dorsiflexion, $1 / 3$ of the subject's phalanges and metatarsal bones were placed onto a wooden block that was $5.5 \mathrm{~cm}$ in height. After a goniometer was used to confirm $20^{\circ}$ of dorsiflexion, subjects were instructed to maximally raise the soles of their feet by standing on their tip-toes, and then to return back into $20^{\circ}$ of ankle dorsiflexion, which was considered as one heel-drop (Figure 1C).

An assistant was available at all times to assist the subjects in maintaining their balance while performing the heel drop exercises at a self-regulated speed. Ten repetitions of each heel drop exercise were considered to be 1 set. A total of 10 sets were performed with a rest period of 30 seconds between each set. Between each of the three dorsiflexion settings for the heel-drop exercises, a 24-hour rest period was provided.

\section{Measurements}

\section{Gastrocnemius muscle thickness and pennation angle}

Subjects were asked to lie prone with ankles off the table during the ultrasound measurement of the gastrocnemius muscle thickness and pennation angle.

Prior to the intervention, the same examiner used a marker pen to enable the exact measurement of the same point on the gastrocnemius muscle immediately after intervention. To optimize the transducer and skin contact, a water-soluble transmission gel was applied prior to the ultrasound scanning process. After the transducer was placed onto the dominant lower extremity behind the knee, between the condyles, $13 \mathrm{~cm}$ inferior from the medial popliteal fossa, ultrasound measurements of muscle thickness and pennation angle was
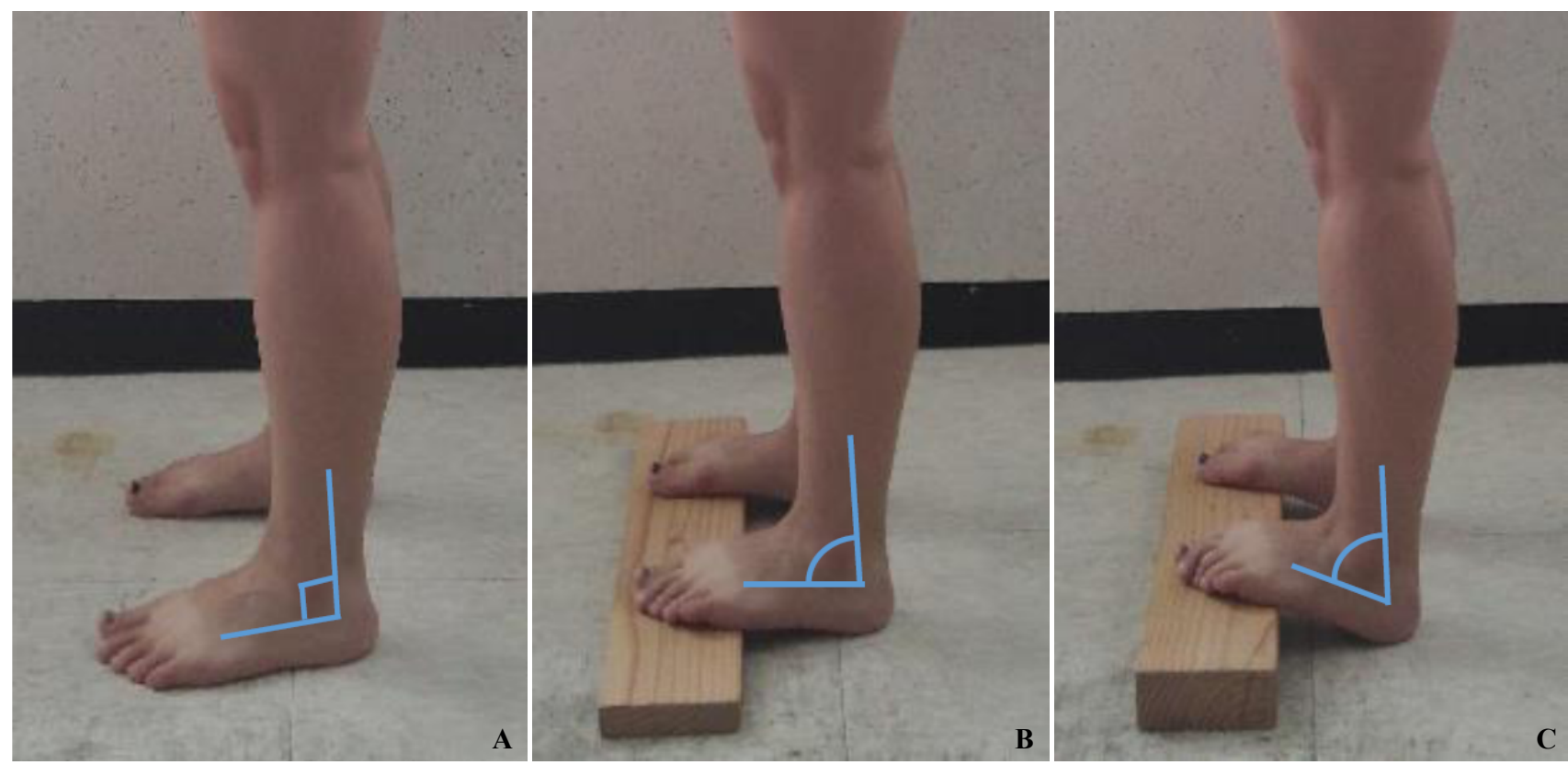

Figure 1. Heel-drop exercise with various degrees of ankle dorsiflexion. (A) $0^{\circ}$ dorsiflexion, (B) $10^{\circ}$ dorsiflexion, (C) $20^{\circ}$ dorsiflexion. 


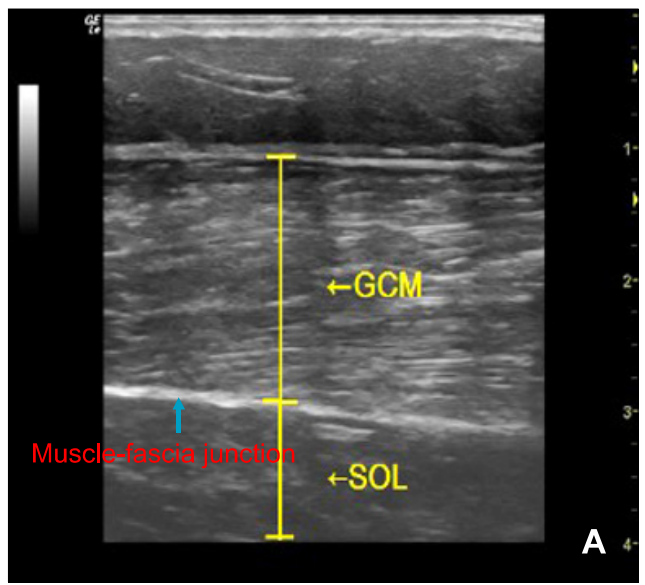

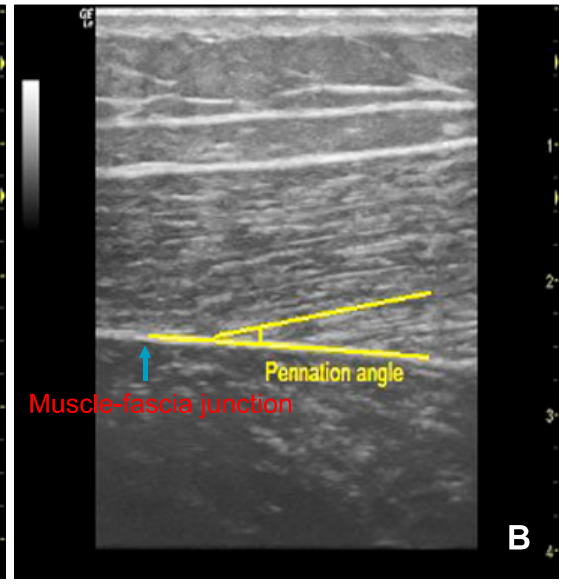

Figure 2. Ultrasonography imaging on the gastrocnemius muscle (GCM). (A) GCM thickness, (B) GCM pennation angle. SOL: soleus.

Table 2. Gastrocnemius muscle thickness post heel-drop exercise

$(\mathrm{N}=19)$

\begin{tabular}{ccccccc}
\hline & Pre-exercise & $0^{\circ}$ dorsiflexion & $10^{\circ}$ dorsiflexion & $20^{\circ}$ dorsiflexion & $\mathrm{F}$ & $p$ \\
\hline Thickness $(\mathrm{cm})$ & $1.74(0.10)$ & $1.96(0.07)^{\mathrm{a}}$ & $2.06(0.08)^{\mathrm{a}, \mathrm{b}}$ & $2.18(0.09)^{\mathrm{a}}$ & 14.24 & 0.000 \\
\hline
\end{tabular}

Values are presented as mean (SD).

${ }^{\mathrm{a}}$ Significant differences compared with pre-exercise $(p<0.05)$. ${ }^{\mathrm{b}}$ Significant difference compared with $0^{\circ}$ dorsiflexion $(p<0.05)$.

Table 3. The pennation angle of gastrocnemius post heel-drop exercise

\begin{tabular}{cccccccc}
\hline & Pre-exercise & $0^{\circ}$ dorsiflexion & $10^{\circ}$ dorsiflexion & $20^{\circ}$ dorsiflexion & $\mathrm{F}$ & $p$ \\
\hline Pennation $\left({ }^{\circ}\right)$ & $8.84(4.07)$ & $9.42(4.88)$ & $6.84(2.93)$ & $8.42(3.53)$ & 2.14 & 0.106 \\
\hline
\end{tabular}

Values are presented as mean (SD).

taken place in the sagittal plane of the gastrocnemius medialis.

Medial gastrocnemius muscle, which is in the outer side of the muscle-fascia, was used to measure the gastrocnemius muscle thickness (Figure 2A), and the pennation angle is the angle created between the muscle-fascia junction and the gastrocnemius fascicle. This angle becomes greater during muscle contraction compared to during a resting period (Figure 2B) [18].

\section{Statistical analysis}

A repeated measures ANOVA was used to analyze the gastrocnemius muscle thickness and pennation angle values according to the various degrees of ankle dorsiflexion during the heel-drop exercises. The Bonferroni post-hoc correction analysis was used to further compare the gastrocnemius muscle thickness according to the various degrees of ankle dorsiflexion. All statistical analysis was performed using the PASW Statistics ver. 18.0 (IBM Co., Armonk, NY, USA) with a significance level set at $\alpha=0.05$.

\section{Results}

\section{The thickness of gastrocnemius muscle on heel-drop}

There was a significant difference in gastrocnemius muscle thickness depending on the various degrees of ankle dorsiflexion ( $p<0.05$; Table 2 ). The Bonferroni analysis showed that compared to the pre-exercise period, there was a significant increase in gastrocnemius muscle thickness after performing heel-drop exercises with $0^{\circ}, 10^{\circ}$, and $20^{\circ}$ of ankle dorsiflexion, with an increase in $22 \mathrm{~mm}, 32 \mathrm{~mm}$, and $44 \mathrm{~mm}$ respectively $(p<0.05)$. There was a significant increase of 1 $\mathrm{mm}$ in gastrocnemius muscle thickness with $10^{\circ}$ versus $0^{\circ}$ of ankle dorsiflexion $(p<0.05)$. However, there was no significant difference in muscle thickness when comparing $10^{\circ}$ and $20^{\circ}$ of ankle dorsiflexion (Table 2). 


\section{The pennation angle of gastrocnemius post heel-drop exercise}

There was no significant difference in pennation angle during heel-drop exercise performance with various degrees of ankle dorsiflexion (Table 3).

\section{Discussion}

Research on rehabilitation methods for those who are at risk for falls as well as development of fall risk prevention interventions for the elderly population are being emphasized.

The gastrocnemius contributes to the calf muscle structure of the lower extremity. Through sustained contraction, the gastrocnemius plays an important role in maintaining the center of mass during gait, and is involved in maintaining stability during the performance of lower extremity exercises [19]. In considering a rehabilitation program for gastrocnemius muscle strengthening, it is important to promote gastrocnemius muscle hypertrophy, function, and strength [20].

The heel-drop exercise used in this study is considered as a stretch-shorten cycle exercise that promoted eccentric strengthening of the gastrocnemius, but it also produced an increase in Achilles tendon elongation and a decrease in tendon stiffness, thus leading to changes in the normal length-tension relationship, and it also served as an indicator for the mechanical fatigue [17]. The aim of this study was to investigate for the effects of heel-drop exercise performance according to various lengths of the gastrocnemius muscle and to compare the strength training effects through ultrasound imaging.

Reid et al. [21] conducted a study where subjects with and without Achilles tendonopathy performed eccentric calf muscle exercises and through the use of electromyography, results had shown that those without Achilles tendonopathy displayed a greater increase in muscle activation compared with those with Achilles tendonopathy. A study by Lee $e t$ al. [22] compared the gastrocnemius muscle thickness through ultrasonography during calf-raise exercises performed on various support surfaces and had found a significant increase in gastrocnemius muscle thickness when performed on all support surfaces. These findings can be related to the results of this study, where all of the normal, healthy subjects had shown improvement in gastrocnemius muscle strength after the performance of heel-drop exercises compared with pre-exercise conditions. A study by Jeong et al. [14] examined the effects of heel-drop exercise performance according to various ankle joint range of motion. The study results showed a significant increase in Achilles tendon stiffness when performing the heel-drop exercise while standing on a block compared to performing the exercise on the floor. Tendon stiffness is influenced by the muscle-tendon complex behavior, which reduces the electromechanical delay and cause an increase in rate of torque development [23]. Considering that fact that torque improvement has the ability to decelerate the body movement and plays a role in fall prevention [24], the results of this study demonstrate similar findings in that that there was a significant increase in gastrocnemius muscle thickness when performing the heel-drop exercise with $10^{\circ}$ ankle dorsiflexion compared with $0^{\circ}$. Heel-drop exercises are considered to be a dynamic eccentric contraction exercise and an eccentric contraction exercise exerts a greater force than isometric contraction exercise. A greater force exertion generates more strain onto the Achilles tendon cells, and if elongation of the tendon occurs, there is an increase in the ability to store energy, which thereby reduces the muscle fiber bundle contraction speed and enhances the power-speed relationship [25].

In this particular study, there were no significant changes in pennation angle during heel-drop exercise performance with use of various degrees of dorsiflexion. Sanz-López et al. [18]. A study by Sanz-López et al. [18] found that after subjects had participated in eccentric overload training and running performance, there was an increase in both cross-sectional area and pennation angle of the gastrocnemius muscle had increased after both interventions. Although generally the pennation angle is associated with muscle hypertrophy and that a greater increase in contraction time develops compared to relaxation time, this could not be proven from the results of this study. The lack of reliability in pennation angle measurement methods and transducer position could be considered as a limitation to this study. Although the subjects participated in the heel-drop exercises in two day intervals in order to prevent to carry over effect, however, its effect could not be ruled out. Another limitation was that although participation in intense sports could have been regulated, the daily activities performed by the subjects could not be controlled. In addition, analysis of heel-drop exercises on gastrocenemius muscle thickness and pennation angle was difficult to prove. Therefore, future studies with exercise designs for heel-drop exercises applied simultaneously for fall prevention in the 
elderly population, Achilles tendon strain reduction, and ankle joint muscle strengthening are needed.

\section{Conflict of Interest}

The authors declared no potential conflicts of interest with respect to the authorship and/or publication of this article.

\section{References}

1. Fuller GF. Falls in the elderly. Am Fam Physician 2000;61: 2159-68.

2. Singh MM, Malhotra HS. Falls in the elderly--clinician's approach. J Indian Med Assoc 2003;101:420, 422, 424 passim.

3. Spirduso WW, Francis KL, MacRae PG. Health, exercise and cognitive function. In: Spirduso WW, Francis KL, MacRae PG, editor(s). Physical dimensions of aging. 2nd ed. Champaign (IL): Human Kinetics; 2005. p. 211-32.

4. Wolfson L, Judge J, Whipple R, King M. Strength is a major factor in balance, gait, and the occurrence of falls. J Gerontol A Biol Sci Med Sci 1995;50:64-7.

5. Lamontagne A, Richards CL, Malouin F. Coactivation during gait as an adaptive behavior after stroke. J Electromyogr Kinesiol 2000;10:407-15.

6. Nam HS, Park DS, Kim DH, Kang HJ, Lee DH, Lee SH, et al. The relationship between muscle fatigue and balance in the elderly. Ann Rehabil Med 2013;37:389-95.

7. Shumway-Cook A, Woollacott MH. Motor control: theory and practical applications. 2nd ed. Maryland: Lippincott Williams \& Wilkins; 2000.

8. Almeida GL, Carvalho RL, Talis VL. Postural strategy to keep balance on the seesaw. Gait Posture 2006;23:17-21.

9. Choi SW, Lee JS, Ku HJ, Lee DT. Effects of resistive and balance training on walking patterns in the falls experienced elderly women. Korean J Phys Educ 2005;44:287-95.

10. Son JC. The effects of ankle strength exercise on the ability of balance control in patients with stroke [Master thesis]. Yongin: Yong In University; 2005.

11. Lee SE. Effects of increasing ankle range of motion program on ambulation and balance for the elderly with balance disorder. Phys Ther Korea 2005:12:28-36.

12. Alfredson H, Pietilä $T$, Jonsson P, Lorentzon R. Heavy-load eccentric calf muscle training for the treatment of chronic Achilles tendinosis. Am J Sports Med 1998;26:360-6.

13. Stevens M, Tan CW. Effectiveness of the Alfredson protocol compared with a lower repetition-volume protocol for midportion Achilles tendinopathy: a randomized controlled trial. J Orthop Sports Phys Ther 2014;44:59-67.

14. Jeong S, Lee DY, Choi DS, Lee HD. Acute effect of heel-drop exercise with varying ranges of motion on the gastrocnemius aponeurosis-tendon's mechanical properties. J Electromyogr Kinesiol 2014;24:375-9.

15. Chi-Fishman G, Hicks JE, Cintas HM, Sonies BC, Gerber LH. Ultrasound imaging distinguishes between normal and weak muscle. Arch Phys Med Rehabil 2004;85:980-6.

16. Fukunaga T, Kawakami Y, Kuno S, Funato K, Fukashiro S. Muscle architecture and function in humans. J Biomech 1997;30:457-63.

17. Obst SJ, Newsham-West R, Barrett RS. Changes in Achilles tendon mechanical properties following eccentric heel drop exercise are specific to the free tendon. Scand J Med Sci Sports 2016;26:421-31.

18. Sanz-López F, Berzosa Sánchez C, Hita-Contreras F, Cruz-Diaz D, Martínez-Amat A. Ultrasound changes in Achilles tendon and gastrocnemius medialis muscle on squat eccentric overload and running performance. J Strength Cond Res 2015. [Epub ahead of print]

19. Youm $\mathrm{CH}$, Kim TH. Effects of induced fatigue of ankle joint muscle on the capability and recovery of postural control during single-leg stance. Korean J Sport Biomech 2012;22:219-28.

20. Arnsdorff K, Limbaugh K, Riemann BL. Analysis of heel raise exercise with three foot positions. Int J Exerc Sci 2011;4:13-21.

21. Reid D, McNair PJ, Johnson S, Potts G, Witvrouw E, Mahieu N. Electromyographic analysis of an eccentric calf muscle exercise in persons with and without Achilles tendinopathy. Phys Ther Sport 2012;13:150-5.

22. Lee GC, Kim B, Kim JS, Nam IS, Park YJ, Shin WJ, et al. The effect of calf-raise exercise on gastrocnemius muscle based on other type of supports. J Korean Soc Intergr Med 2014;2:109-16.

23. Reeves ND, Maganaris CN, Narici MV. Effect of strength training on human patella tendon mechanical properties of older individuals. J Physiol 2003;548:971-81.

24. Carville SF, Perry MC, Rutherford OM, Smith IC, Newham DJ. Steadiness of quadriceps contractions in young and older adults with and without a history of falling. Eur J Appl Physiol 2007; 100:527-33.

25. Fletcher JR, Esau SP, MacIntosh BR. Changes in tendon stiffness and running economy in highly trained distance runners. Eur J Appl Physiol 2010;110:1037-46. 